\title{
How Random Is Spatiotemporal Chaos of Langton's Ant?'
}

\author{
I. DIRGOVÁ LUPTÁKOVÁ I. AND J. POSPÍCHAL
}

\begin{abstract}
In recent years there have been numerous attempts to control chaotic behavior by evolutionary optimization. Most of these attempts were aimed at a study of chaotic systems defined by differential equations, but a few attempts were made also at evolutionary design of initial conditions or rules of cellular automata aimed at performing a specified task. We shall use a simple cellular automaton called Langton's ant after its designer, Christopher Langton. Generally, the ant acts on a 2D grid, where each it's square can be either black or white. The ant is facing in one of four directions, and its behavior is described by 3 rules: (1) If ant is on a black square, it makes a left turn. (2) If ant is on a white square, it makes a right turn. (3) When ant moves to the next square, the one it was on reverses color. Despite simplicity of these rules, the ant produces extremely complex behavior, but after around 10000 steps the ant begins to construct a diagonal ,highway“. This stable attractor has been always achieved regardless of the initial setting of black and white squares, but there is no proof, that it is always so. This behavior can be related to the undecidability of the halting problem. Our goal in this paper is to optimize initial conditions for the ant on a grid, so that it will be maximally "slowed down" in the sense that it should arrive at the preset boundary of the grid as late as possible. By a comparison of greedy stochastic optimization with an optimization by blind search are able to estimate, that is this chaotic system is not reasonably controllable and appears to have no regularity in the "optimal" initial conditions.
\end{abstract}

Mathematics Subject Classification 2000: 37B15, 34C28, 68W20

General Terms: cellular automata, chaotic behavior, optimization

Additional Key Words and Phrases: Langton's ant, Turing machine

\section{INTRODUCTION TO CELLULAR AUTOMATA AND LANGTON'S ANT}

Cellular automata are discrete dynamic systems, used typically in simulations ranging from fluid dynamics or galaxy formation up to crowd control (MITCHELL et al., 1994; SCHIFF 2008; FENG et al., 2011). Their massive parallelism and resistance to noise make them also suitable for engineering applications like imageprocessing task (ROSIN, 2006). Apart from practical applications, cellular automata are also popular for studying discrete chaos theory (MARTINEZ et al., 2012).

In recent years there have been numerous attempts to control chaotic behavior by evolutionary optimization. Most of these attempts were aimed at a study of chaotic systems defined by differential equations, but quite a few attempts were

\footnotetext{
${ }^{1}$ Acknowledgement: This contribution was supported by Grant Agency VEGA SR under the grant $1 / 0458 / 13$. 
made also at evolutionary design of initial conditions or rules of cellular automata aimed at performing a specified task (MITCHELL et al., 1994; FENG et al., 2011).

Apart from the most popular two-dimensional cellular automaton Conway's Game of life, one of the interesting two-dimensional cellular automata is a universal Turing machine called Langton's ant. It was proposed by Langton (LANGTON, 1986), and its theoretical properties like ability to perform logical calculations were further studied e.g. in (GAJARDO et al., 2002). Typically, the ant acts on a twodimensional square lattice, where each square can be either black or white. The ant normally starts on a white lattice and is facing in one of four directions. Its behavior is described by 3 rules: (1) If ant is on a black square, it makes a left turn. (2) If ant is on a white square, it makes a right turn. (3) When ant moves to the next square, the one it was on flips its color from black to white or from white to black.

A further extension of Langton's ants can use multiple states of the Turing machine (it is then called turmite as a contraction of "Turing machine termites", see PEGG, 2015), and/or hexagonal lattice can be used, together with a study of interaction of several ants on the same lattice (SCHIFF 2008).

\section{TOTAL SEARCH FOR BEST INITIAL CONDITIONS WITH ONE AND TWO BLACK CELLS}

The ant typically "wanders" around, generating chaotic irregular pattern of black and white squares, until it starts to create a diagonal "highway", produced by a recurrent pattern of 104 steps that repeats indefinitely (in chaos terms this "highway" is an attractor, towards which the ant converges). While Cohen-Kong theorem (STEWART, 1994) proved, that the ant always crosses any preset boundary, the inevitability of production of this "highway" from any finite initial configuration is confirmed only experimentally. In our paper, we study an influence of an initial pattern of black cells in a lattice on a final pattern produced by the ant. In order to estimate this influence, we have placed an ant in the center of a square lattice of $201 \times 201$ white cells, faced the ant towards the top and blackened one cell in the central 11x11 part of square lattice. Then we let the ant wander around, until it 
reached the border of the lattice. The number of ant's steps ranged from 5122 to 49570; these two patterns are shown in Figure 1.

A:

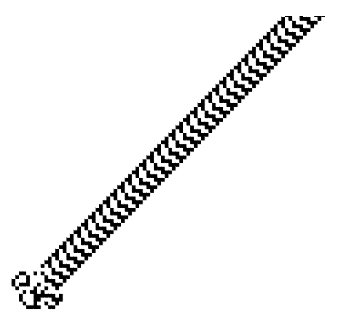

B:

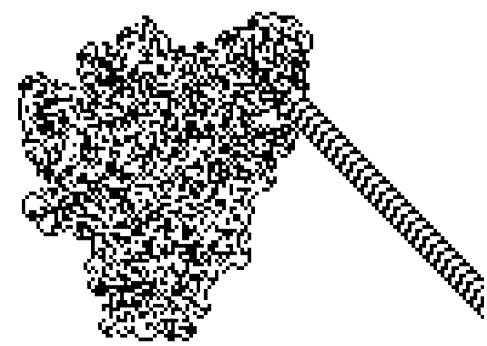

Fig. 1. The figures show sensitivity of the automaton to initial conditions. The figure A was produced by Langton's ant, when a single black cell was positioned 3 rows down and 3 columns right from the initial position of the ant, the figure B was produced by shifting this additional black cell one position to the right.

Figure 2 shows positions of the one added blacked cell, shaded according to the number of steps which the ant used to reach a border, as it is shown on the scale to the right. No pattern can be found to estimate, which initial position would cause most/least steps.

Since there was no pattern which would emerge by adding one black cell into a central part of an initial square, we tried adding two black squares, one positioned, so that it would otherwise produce the maximum 49570 steps, when added as single square, and a second black square positioned in all other possible places within the central 11x11 part of square lattice. The question was: If the second square was positioned into a position, which produced a high number of steps when it was a single square, would it increase the number of steps when added to the previous optimal position? If this was the case, if we would order the cells by the increasing number of produced steps on horizontal axis, and similar order was used for a vertical axis, only with added "maximum cell", we should ideally get a linear correlation - line going from the bottom left to the top right. Figure 3 shows that clearly no such correlation exists, so that we cannot from a suitable position of one cell predict a suitable position of an added second cell. 


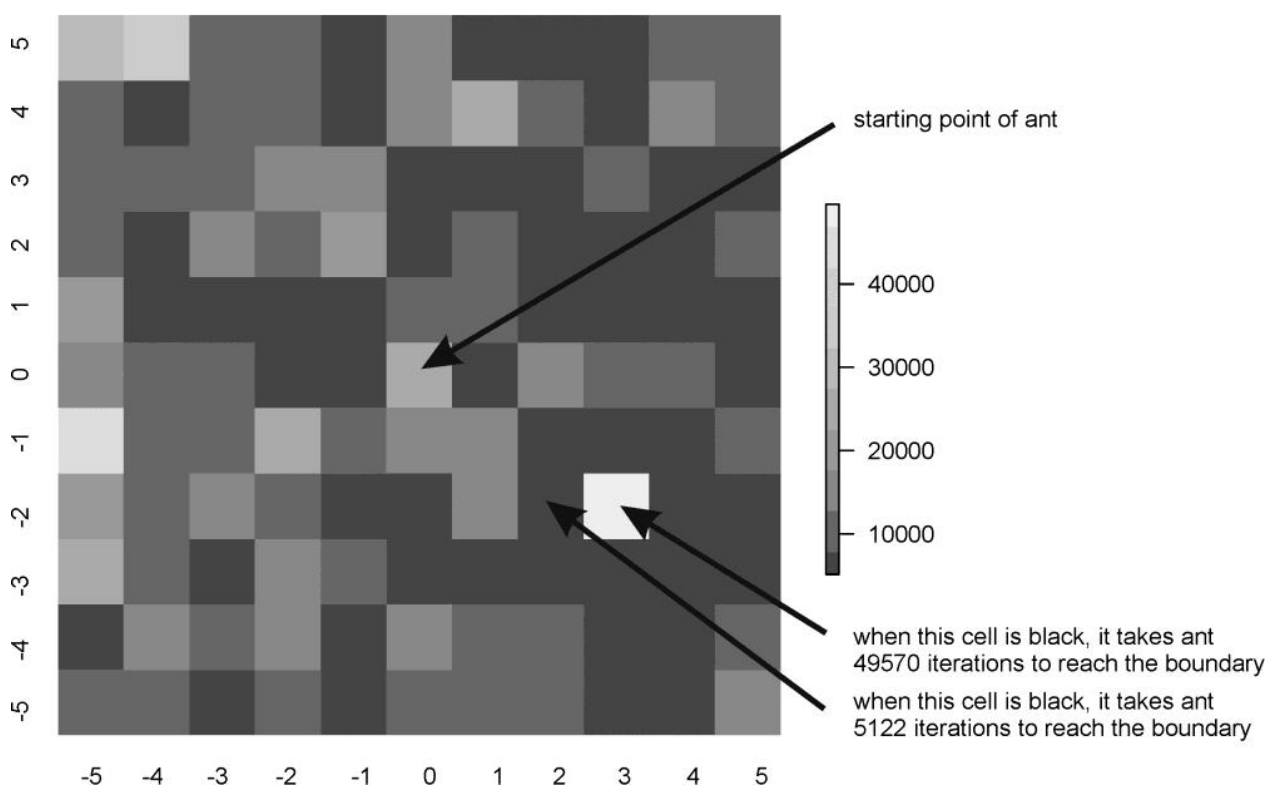

Fig. 2. The diagram shows the randomness of Langton's ant response caused by a change of its initial condition by adding one black cell into different positions ranging from -5 to 5 cells horizontally or vertically from the initial position of the ant.

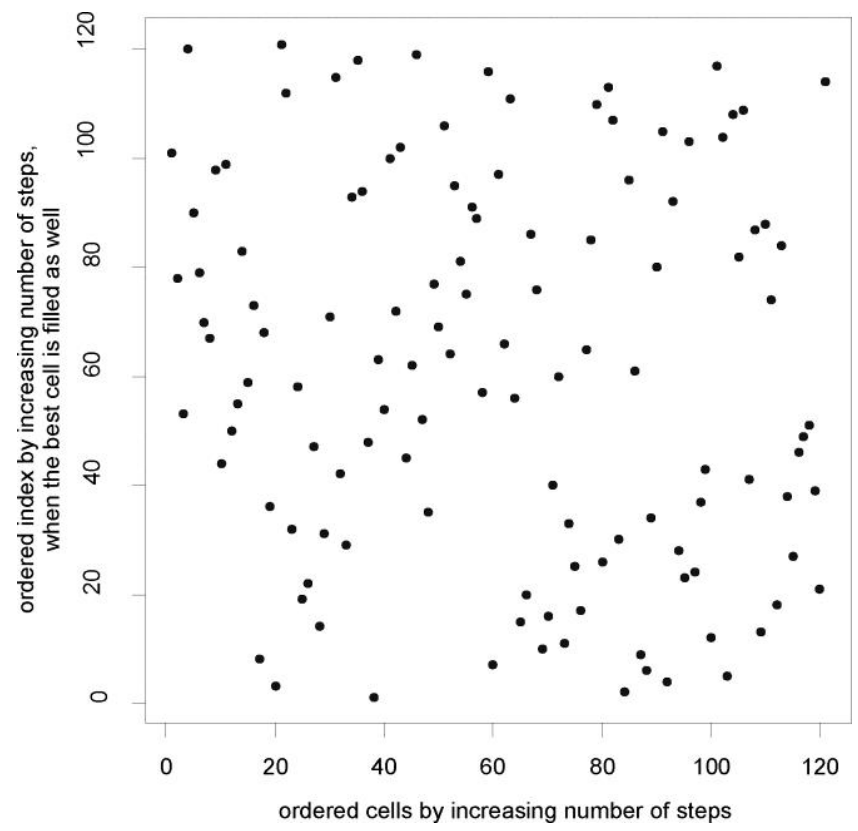


Fig. 3. The diagram shows the randomness of Langton's ant response caused by a change of its initial condition by adding two black cells into different positions ranging from -5 to 5 cells horizontally or vertically from initial position of the ant.

\section{HILL-CLIMBING AND BLIND RANDOM SEARCH OPTIMIZATION}

Since a total search of most simple cases failed to produce anything useful for prediction of a pattern producing greatest number of steps, we tried to optimize the initial pattern by a classical hill-climbing algorithm. This means, that we found the position of a single cell black cell in the central $11 \times 11$ square producing most steps, fixed it and found a second cell, which produced together with the first one most steps etc. However, we soon reached a suboptimum, when no single added blackened cell added to the previous initial pattern increased the number of steps. Therefore we tried a randomized hill-climbing, see Algorithm 1, when we randomly flipped 5 cells in the central $11 \times 11$ square between black and white, and if these initial conditions produced a higher number of steps, we kept these initial conditions as a starting points and continue flipping 5 randomly chosen cells in the central $11 \times 11$ square. By flipping we mean, that previously black cell also could become white. This we repeated for 100000 iterations and statistics was collected for 100 runs.

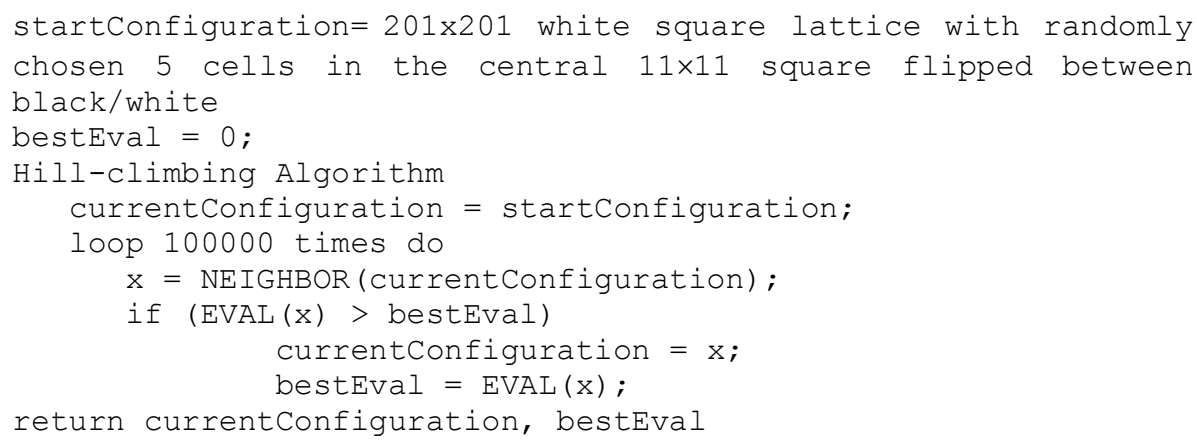

Algorithm 1. Hill-climbing optimization of initial configuration of a two-dimensional square black/white lattice. Function NEIGHBOR flips between black/white 5 randomly chosen cells in the central $11 \times 11$ square, function EVAL counts number of steps the ant needs to reach the border of the $201 \times 201$ lattice 
As it can be seen from Figure 4, we achieved for the best found pattern more than 220000 steps, more than four times higher number compared to full search optimization of a single cell position. Superficially, it appears that such a randomized greedy optimization works very well. However, it is always suitable to compare a proposed algorithm with a competing one.

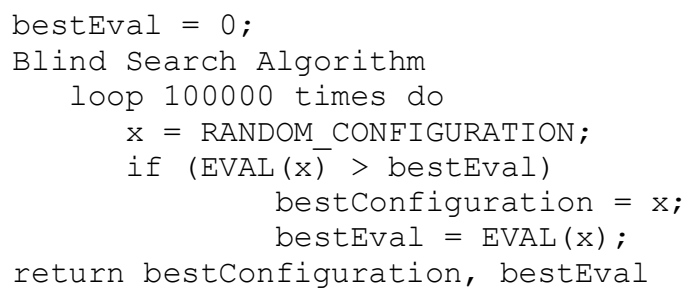

Algorithm 2. Blind search optimization of initial configuration of a two-dimensional square black/white lattice. Function RANDOM_CONFIGURATION takes 201x201 white square lattice and changes from white to black 35 randomly chosen cells in the white central $11 \times 11$ square, function EVAL counts number of steps the ant needs to reach the border of the $201 \times 201$ lattice 


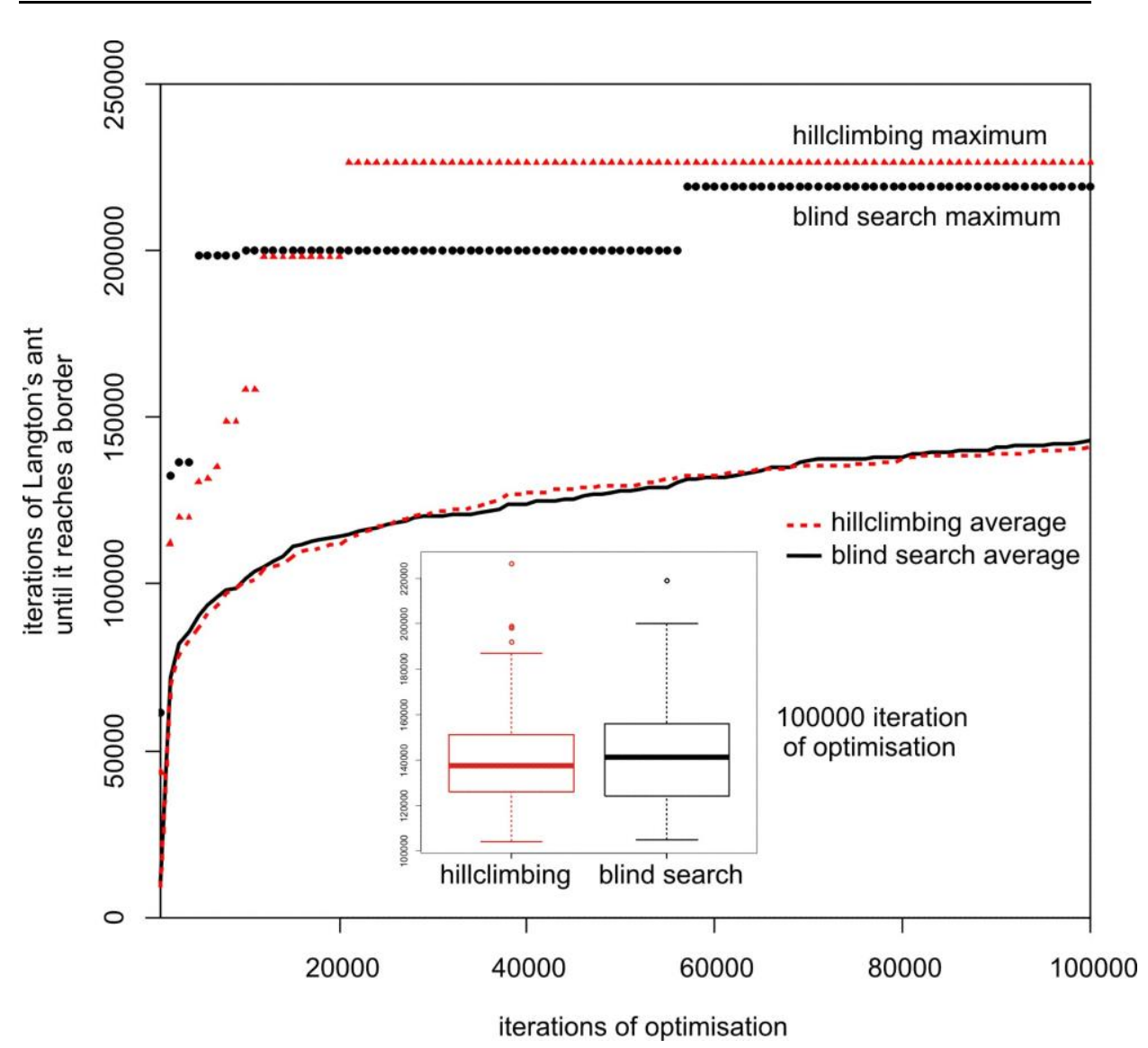

Fig. 4. Comparison of optimizing initial conditions by hill-climbing and by blind search. The central box plot shows the statistics from 100 runs at the 100000 iteration. Clearly, none of the optimization approaches has an advantage over the other.

We have chosen a most primitive optimization algorithm - a "blind search" in our implementation an uninformed random search, see Algorithm 2, when we simply repeatedly randomly selected some cells within the central $11 \times 11$ square to be initially black, run the ant to measure how many steps it would produce and stored the greatest number of steps together with the best randomly generated initial pattern. 
Since at the end of optimization the best initial conditions had 37 blackened cells, we tried to compare the hill-climbing with a blind search procedure, where 35 cells were randomly blackened.

Unfortunately, the Figure 4 shows that the blind random search is fully comparable with hill-climbing, which suggests, that hill-climbing heuristic was unable to find any correlation between initial cells or any hidden structure in the optimized initial conditions. In the Figure 4 you can see a graph of average number of steps of the ant on vertical axis against the number of iterations of hill-climbing (dashed red line) or blind random search (solid black line) on horizontal axis. The maximum values from the 100 runs can be found above these lines, red triangles for hill-climbing and black dots for blind random search. The distribution of the results after 100000 iterations of an optimization algorithm is shown in the box plot showing interquartile range as a box with median as thick line, whiskers showing lowest datum still within 1.5 IQR of the lower quartile, and the highest datum still within 1.5 IQR of the upper quartile, and circles presenting outliers. Both algorithms produce very similar results.

\section{CONCLUSIONS}

It was shown, that initial condition of the cellular automaton Langon's ant cannot be easily optimized and stochastic hill-climbing does not provide better results in this case than the most basic random blind search. On the other hand, this result should provide a support for applications of Langon's ant, which actively use chaos and pseudorandom quality of the pattern of black and white cells produced by the ant, like image encryption (WANG, 2015) or pseudorandom number generation (HOSSEINI, 2011).

\section{ACKNOWLEDGMENTS}

This contribution was supported by Grant Agency VEGA SR under the grant $1 / 0458 / 13$ 


\section{REFERENCES}

FENG, Y., LIU, Y., TONG, X., LIU, M., AND DENG, S. 2011. Modeling dynamic urban growth using cellular automata and particle swarm optimization rules. Landscape and Urban Planning 102(3), 188196.

GAJARDO, A., GOLES, E., AND MOREIRA, A. 2002. Complexity of Langton's Ant. Discrete Applied Mathematics 117, 41-50.

HAMANN, H., SCHMICKL, T., AND CRAILSHEIM, K. 2011. Thermodynamics of emergence: Langton's ant meets Boltzmann. In: IEEE Symposium on Artificial Life (ALIFE), April, 2011, 62-69.

HOSSEINI, S. M., KARIMI, H., AND JAHAN, M. V. 2011. From Complexity to Random Behaviors; Generate Random Numbers by Confusion in Cellular Automata State's. In ARABNIA H. R. AND GRAVVANIS G. A. (Eds.): CSC'11, The 2011 International Conference on Scientific Computing, Las Vegas, Nevada, USA, July, 2011, pp. 262-268.

LANGTON, C. G. 1986. Studying artificial life with cellular automata. Physica 22D 120-149.

MARTINEZ, G. J., ADAMATZKY, A., AND ALONSO-SANZ, R. 2012. Complex dynamics of elementary cellular automata emerging from chaotic rules. International Journal of Bifurcation and Chaos 22(02), 1250023.

MITCHELL, M., CRUTCHFIELD, J. P., AND HRABER, P. T. 1994. Evolving cellular automata to perform computations: mechanisms and impediments. Physica D: Nonlinear Phenomena 75, 361-391.

PEGG, E. Jr.: "Turmite". From MathWorld--A Wolfram Web Resource, created by Eric W. Weisstein. [online]. [cit. 2015-08-09]. Available on internet: http://mathworld.wolfram.com/Turmite.html

ROSIN, P. L. 2006. Training cellular automata for image processing. IEEE Transactions on Image Processing 15(7), 2076-2087.

SCHIFF, J. L. 2011. Cellular automata: a discrete view of the world. John Wiley \& Sons, Hoboken, NJ, USA.

STEWART, I. 1994. The Ultimate in Anty-Particles. Sci. Amer. 271, 104-107.

WANG, X., AND XU, D. 2015. A novel image encryption scheme using chaos and Langton's Ant cellular automaton. Nonlinear Dynamics 79(4), 2449-2456.

\section{Iveta Dirgová Luptáková}

Department of Applied Informatics and Mathematics, University of SS. Cyril and Methodius, Trnava, SK-917 01, Slovak Republic, iveta.dirgova@ucm.sk

\section{Jiří Pospíchal}

Department of Applied Informatics and Mathematics, University of SS. Cyril and Methodius, Trnava, SK-917 01, Slovak Republic, jiri.pospichal@ucm.sk

Received October 2015 